McElroy, T. \& Mascari, D. (2007). When is it going to happen? How temporal distance influences processing for risk-choice framing tasks. Social Cognition, 25(4): 495-517. Published by Guilford Press (ISSN: 0278-016X). Copyright Guilford Press. Reprinted with permission of The Guilford Press.

\title{
When Is It Going To Happen? How Temporal Distance Influences Processing for Risky-Choice Framing
}

\section{Tasks}

Todd McElroy and Dominic Mascari

\author{
ABSTRACT \\ In this article we examine how temporal proximity of an event influences \\ decision task processing and, in turn, the likelihood of framing effects. We \\ hypothesized that events occurring in the relatively near future should be \\ more likely to induce the analytic processing style and result in attenuated \\ framing effects. Events occurring in the more distant future should be processed \\ with the holistic style and framing effects should be relatively more \\ pronounced. In Study 1, we assessed how temporal proximity influenced \\ the perceived relevance for an event occurring at different temporal \\ proximities. In Study 2, we tested the influence of temporal proximity using \\ the Asian disease problem. In Study 3, we tested the influence of temporal \\ proximity with a different type of risky-choice decision task and investigated \\ evidence for analytic/holistic processing. The results provide \\ converging support for a temporal-relevance hypothesis.
}


Over two decades of research has investigated the finding that presentation of alternatives, as positive or negative outcomes, will substantially influence a decision-maker's choice. It stands to reason that this change of preference, based solely on presentational frame, does not follow a logical or rational course. Rather, most people agree that preference for one option over another should remain constant regardless of the frame in which the options are presented. The most widely investigated example of this change in preference has become known as the framing effect.

Kahneman and Tversky (1979) first depicted this effect in the development of prospect theory. According to prospect theory, the value function is not linear and differs for gains and losses as depicted in the S-shaped value function. For both gains and losses, the value curve is negatively accelerating yielding a concave curve for gains and a convex curve for losses. A direct prediction from prospect theory is that, when dealing with problems that are framed in terms of gains, individuals should tend to be relatively risk averse, whereas when the problem is framed in terms of losses, they should tend to be risk seeking. It is from this prediction that work on framing effects has evolved.

Since the introduction of prospect theory, the framing effect has become the most widely tested example of irrational decisionmaking. Although mostly supportive, some research investigating framing effects has demonstrated inconsistencies, including both null effects and "preference reversals" (see Kühberger, 1998 and Levin, Schneider, \& Gaeth, 1998 for reviews). Such inconsistent findings have led researchers to further investigate person and situational factors that can influence the likelihood of framing.

Research has revealed that contextual factors, such as requests for elaboration (Takemura, 1994; LeBoeuf \& Shafir, 2003; Miller\& Fagley, 1991; Sieck \& Yates, 1997), context indicators (Bless, Betxch, \& Franzen, 1998), target of the task (Levin \& Chapman, 1990; Wang, 2001), and presentation format (Fagley \& Miller, 1997) can affect the likelihood of framing. It is also the case that person factors such as need for cognition (Chatterjee, Heath, Milberg, \& France, 2000; Curseu, 2006; Smith \& Levin, 1996; Zhang \& Buda, 1999; Simon, Fagley, \& Halleran, 2004), intuition (Levin, Gaeth, \& Schreiber, 2002), competence (Bruine de Bruin, Parker, \& Fischoff, 2007), and predispositions toward analyticaldeliberative thought (Bartels, 2006; LeBoeuf \& Shafir, 2003, McElroy \& Seta, 2003) appear to play a role in processes involved in the framing effect. 


\section{DUAL-PROCESSING APPROACH}

Theoretical models of dual-processing have proven effective in many areas of research (e.g., Epstein, Lipson, Holstein, \& Huh, 1992; Chaiken, 1987; Petty \& Cacioppo, 1986; Stanovich \& West, 2000) but have proven especially important for social psychology (Chaiken \& Trope, 1999). At the heart of the dual-processing approach lays the belief that processing of information occurs via two fundamentally different routes. Normally, one route is considered to involve more in-depth processing and the other, less in-depth processing.

Research investigating the effects of dual-processing in framing tasks has found that different levels of processing have substantially different effects on participants' choices. While there are differences in the functionality of the two systems, there is general agreement that the "system" the individual relies on plays an important role in how the framing task is processed and subsequent decisions are made (e.g., Bartels, 2006; Igou \& Bless, 2007; Leny-Meyers \& Maheswaran, 2004; Reyna \& Brainerd, 1991; McElroy \& Seta, 2003; Wang, 2006)

\section{ANALYTIC/HOLISTIC MODEL}

According to the analytic/holistic model, two systems of processing operate independently and under different principles. The model identifies three factors that can determine which processing style will be more or less likely to be used. Specifically, research investigating the model has demonstrated that relevance (McElroy \& Seta, 2003; 2006), personality predisposition (McElroy \& Seta, 2003), and hemispheric activation (Gallagher \& Dagenbach, 2007; McElroy\&Seta, 2004) can all act as determining factors for the processing style individuals rely upon.

Although applicable to other decision tasks, research testing this model has focused on gaining a better understanding of the framing effect. In one study, McElroy and Seta (2003) demonstrated that tasks of sufficiently high personal relevance induced the more effortful/analytic style, leading to greater numeric sensitivity and little or no framing influence. Tasks of low personal relevance induced the less effortful/holistic processing style, leading to heightened sensitivity to contextual cues and greater influence of framing. Later research has supported the relevance principle of this model (Leny-Meyers \& Maheswaran, 2004; McElroy \& Seta, 2006).

Situational factors also can act to induce either processing style via relative hyperactivation of the respective hemispheres. Conditions that lead to relative hyperactivation of the left hemisphere induce more analytic processing and less framing whereas 
hyperactivation of the right hemisphere induces the holistic processing style, leading to relatively stronger framing effects (McElroy \& Seta, 2004).

Similar to other dual-process models, the analytic/holistic model incorporates the effort (e.g., Chaiken, 1987; Petty \& Cacioppo, 1986) and personality (e.g., Epstein, Lipson, Holstein, \&Huh, 1992) principles seen in other dual-process models. However, it also includes the functional specializations of the respective hemispheres as a means for inducing the respective processing styles (McElroy \& Seta, 2004).

The analytic/holistic model differs from other dual-processing models in how the two styles process information. The analytic system focuses on dismantling the information, breaking it down into distinct elements and then combining the information and focusing on quantitative weights for solutions. This type of processing is characteristic of how information is analyzed in the left hemisphere and leads individuals who are using this processing style to be especially reliant upon the expected value of the options and not on the way in which they are framed. Because both options in a risky-choice framing task have the same expected value (or numeric magnitude) ideally there should be no preference between the options. The expected value of an outcome, however, typically is not objective but rather subjective. There is often, for example, a premium placed on an outcome that will be obtained with certainty (e.g., Machina, 1982; Slovic \& Lichtenstein, 1968). Thus, although the preference of individuals using an analytic processing style is resistant to the influence of a frame, it may still be influenced by the subjective value that individuals assign to the components of the decision problem, such as certainty.

The holistic system relies upon cues from the environment for processing; making it especially sensitive to context. According to the analytic/holistic model, this system operates through a process known as "contextual referencing" (e.g., Brownell, Pincus, Blum, Rehak, \& Winner, 1997). In contextual referencing, context cues elicit a cognitive framework within which the decision problem is interpreted and evaluated and is characteristic of how information is processed in the right hemisphere. Because this processing style relies heavily upon contextual cues (i.e., frames) individuals utilizing this processing style should be especially prone to the influence of the frame, appearing risk-averse for positive frames and risk-seeking for negative frames (Kahneman \& Tversky, 1979). 


\section{RELATED TEMPORAL RESEARCH}

There is a wide array of evidence showing that events occurring at relatively closer temporal proximities are processed differently than events occurring in more distant proximities (Sanna \& Chang, 2006). For example, Gilovich, Kerr, and Medvec (1993) measured participants' confidence about their performance and found that confidence was markedly reduced from the beginning to the end of the semester. Sanna, Chang, Parks, and Carter (2005) investigated how "temporal framing" would influence the group planning fallacy. They found that when individuals were told that there was little time remaining on their task (close temporal proximity) the group planning fallacy was eliminated. There is also evidence that presenting events in more distant temporal proximity may attenuate a fallacy. Malkoc, Zauberman, and Ulu (2005) found that when events were presented in the distant future overreliance on alignable elements of the alternatives was reduced. Moreover, Sanna and Schwarz (2004) found that temporal factors can affect the accessibility of thoughts related to success or failure.

Research investigating temporal construal theory has shown that when events are presented in the relatively distant future individuals appear to make more global attributions, focusing on broader, superordinate implications (Liberman \& Trope, 1998) and often construe the event in more abstract terms (Trope \& Liberman, 2003). For example, in one recent study Liberman, Sagristano, and Trope (2002, Study 2) provided individuals with the task of evaluating the valence (good or bad) of a particular day. The day was presented as occurring in either the relatively distant or near future. They found that when the day was in the near future individuals reported more diversity in terms of the days valence. However, when the day was to occur in the distant future, there was less variance in the valence of the day which resulted in a more prototypical "good day" or "bad day."

Therefore, research investigating the effects of temporal proximity has found that when events are presented as occurring at more distant temporal proximities, individuals are more susceptible to fallacies (Sanna, Chang, Parks, \& Carter, 2005), valenced information (Liberman, Sagristano,\&Trope, 2002) and optimistic bias (Gilovich, Kerr, \& Medvec, 1993) relative to when events are occurring at close temporal proximity. Although the aforementioned research did not directly investigate the relevance of a decision task, it does nonetheless provide evidence that temporal proximity of the event can lead to different processing strategies. 


\section{TEMPORAL PROCESSING AND FRAMING}

Research investigating temporal factors has not directly examined how temporal proximity will influence task relevance. However, we propose that events occurring at more distal temporal points will be perceived as less relevant whereas events occurring at more proximal points will be perceived as more relevant. We use this temporal relevance assumption to make predictions about when framing effects are likely to occur.

According to the analytic/holistic model for framing (e.g., McElroy \& Seta, 2003) the relevance of an event functions to determine which processing style will be induced and, consequently, the likelihood of framing effects. Therefore, because events occurring in close temporal proximity should be perceived as more relevant, they should also be processed using the more effortful, analytic processing style. Consequently, under these conditions, decision-makers should rely on the numeric magnitude of the alternatives and attenuated framing effects should occur. However, if the individual perceives the event as occurring in relatively distant temporal proximity they should perceive the event as less relevant and be more likely to use the less effortful, holistic processing style resulting in more robust framing effects.

\section{OVERVIEW OF STUDIES}

We conducted three studies to examine the relationship between temporal proximity and the framing effect. First, we wanted to test our assumption that events occurring in closer temporal proximity are more relevant than events occurring at a more distant proximity. We designed Study 1 to directly assess this assumption in a risky-choice type decision task. Studies 2 and 3 were designed to examine whether events occurring at different temporal proximities will elicit different processing styles and consequently, influence the likelihood of framing effects. In Study 2 , we experimentally manipulated the future temporal distance at which the Asian disease was to occur and measured its effect on participants' choice preference. In Study 3, we manipulated the temporal proximity of a risky-choice decision task involving cholesterol intake. We then measured participants' choice preference and responses to a frame recall task to assess holistic processing.

\section{EXPERIMENT 1}

\section{METHOD}

Participants. The 40 participants in the study were undergraduate students at Appalachian State University who volunteered to 
participate. Of our participants, 29 were women and 11 were men.

Procedure. After consenting to take part in our study, participants were presented with the stimuli material and asked to consider each of the questions. All of the participants were first given the introduction to the traditional Asian disease problem (seeAppendix 1). They were then asked to rate the relevance of the event if it were to be occurring at four different points in the future (one week, one year, three years, 30 years).1 A 10-point Likert scale ranging from 1 (not relevant) to 10 (extremely relevant) was used to assess participants' reported relevance. The order of the temporal points was counterbalanced between participants. After completion, participants were informed of the purpose of the study and thanked for their participation.

\section{RESULTS AND DISCUSSION}

We first calculated the average relevance scores for each of the temporal points. Participants reported a distinct pattern of relevance with the level of relevance decreasing as the event grew farther away in time (see Table 1). This pattern is consistent with our reasoning that events occurring in relatively closer temporal proximity are seen as more relevant than those occurring in more distant temporal proximity. To investigate these findings, weperformed a one-wayANOVAwith temporal proximity acting as independent variable, and participants reported relevance as our dependent variable. This analysis revealed a significant effect $F$ $(1,148)=29.95, p<.001$. Further, a repeated measures analysis of our data revealed a very similar effect $F(3,37)=26.73, p<.001$. To further investigate this finding we performed contrasts for each of the increasing levels of temporal proximity. These analyses revealed significant increases in relevance from the week to year condition $F(1,148)=8.08, p<.01$, the 1 -year to 3 -year condition $F$ $(1,148)=10.14, p<.01$ and 3 -year to 30-year condition $F(1,148)=$ $8.4, p<.01$.

TABLE 1. Mean Level of Reported Relevance as a Function of Temporal Proximity

\begin{tabular}{lr} 
Temporal Proximity & Average Relevance Score \\
\hline 1 week & 9.13 \\
1 year & 7.9 \\
3 years & 6.5 \\
30 years & 5.2 \\
\hline
\end{tabular}


The results from this study provide empirical evidence that events occurring closer in temporal proximity are seen as more relevant whereas those occurring in more distant temporal proximity are seen as less relevant. As substantial research has shown, more relevant events induce more effortful processing whereas less relevant events induce less effortful processing (e.g., Chaiken, 1987; Petty \& Cacioppo, 1986). Additionally, research has also shown that framing effects are very pronounced when less effortful processing is induced and attenuated when more effortful processing is induced (Leny-Meyers \& Maheswaran, 2004; McElroy \& Seta, 2003; 2006). Therefore, based upon these findings, Experiment 2 was designed to test whether different temporal distances for the Asian disease problem would lead to different types of processing for a framing task and, in turn, differences in the strength of the framing effect.

\section{EXPERIMENT 2}

\section{METHOD}

Participants and Design. The study was made up of 344 undergraduate students; our sample consisted of 194 females and 150 males. The design of our study consisted of a 2 frame (gain, loss) $\times$ 4 temporal proximity (week, 1yr, 3yrs, 30yrs) between factors design.

Procedure. After consenting to participate in our study, all participants were presented with the vignette of the Asian disease problem framed either as gains (saved) or losses (die). Imbedded within the vignette was the indicator of temporal proximity. Participants were presented with one of the four temporal proximity conditions. The different temporal conditions are presented parenthetically below:

Imagine that the U.S. is preparing for the outbreak of an unusual Asian disease. The disease is expected to hit in (a week, one year, three years, thirty years) and kill 600 people. Two alternative programs to combat the disease have been proposed. Assume that the exact scientific estimates of the consequences of the programs are as follows:

Directly afterward, participants were provided with the traditional risk-free, risk-seeking choices. After participants had made their decision, they were debriefed, thanked for their participation, and dismissed from the study.

\section{RESULTS AND DISCUSSION}

We first wanted to test whether the typical gain/loss framing effect appeared across framing conditions. When we collapsed 
across temporal conditions, a strong framing effect was evident $X^{2}$ $(1, N=344)=24.1, p>.0001$. In order to examine our temporal-relevance hypothesis we wanted to test whether temporal proximity of the event influenced processing of the decision task and, in turn, influenced the frame. In order to examine this we performed an analysis to test for framing effects in each of the temporal distance conditions. As can be seen in Table 1, analysis of the one-week condition did not reveal a significant effect for gain/loss framing $X^{2}(1, N=86)=2.9, p<1$. In the one year $X^{2}(1, N$ $=86)=4.7, p<.05$ and three year condition $\times 2(1, N=86)=6.5, p<$ .01 however, significant framing effects were found. The data for the thirty-year condition revealed a very strong framing effect $X^{2}$ $(1, N=86)=12.7, p>.0004$.

The results of this study provide some support for our temporal relevance hypothesis in that framing effects were found when the event was to occur at a relatively distant point in time (i.e., one year or three years) but not when it was to occur at a relative close point in time (i.e., one week). Furthermore, the framing effect was most pronounced when the event was expected to occur at the most distal point in time (i.e., 30 years). These results however, are relatively weak in that the difference between the strength of the framing effect in the one week condition did not differ significantly from the one $\left(X^{2}<1\right)$, three $\left(X^{2}<1\right)$ or thirty year condition $X^{2}$ $(1, N=172)=2.6, p<.11$. It is interesting to note that although there was not a significant difference between the 30 week and one week conditions, there was a descriptive difference $X^{2}(1, N=$ 86) $=3.2, p<.08$ between these two conditions when the problem was framed as a loss, but not as a gain $X^{2}<1$. The relatively strong effect found in the loss frame may be due to differences in the relevance of the Asian disease to participants across these two different frames. Specifically, the negative implications of the Asian disease may have been more relevant to participants when the disease was imminent (i.e., one week) and framed as a loss compared to when it was imminent and framed as a gain. If so, then stronger effects would be expected when the problem was framed as a loss.

\section{EXPERIMENT 3}

Experiment 2 demonstrated some support for our hypothesis that because proximal events are more relevant than distal ones, they are processed more effortfully, leading to attenuated framing effects. The results of Experiment 2, however, were weak. Thus, we wanted to further examine our temporal relevance hypothesis. In Experiment 3, we again manipulated the temporal proximity of an event; one involving cholesterol levels. However, to increase power, we also made changes to our independent variable. Recall that in Experiment 2 the most "imminent" event was to occur in one week. In Experiment 3, we increased the immediacy of the 
event from one week to several hours. We also made changes to our dependent variable. In Experiment 2, we used a dichotomous choice measure. Participants were asked to choose between two different options. Dichotomous measures are typically less powerful than continuous ones. Thus, in Experiment 3, we used a 7-point Likert scale where 1 indicated a definite preference for the risk-averse option and 7 indicated a definite preference for the risk-seeking option (Levin et al., 2002). Finally, we used a memory task to provide a relatively direct test for whether participants used different processing styles across the proximal and distal framing conditions.

TABLE 2. Number of Risk-Avoidant and Risk-Seeking Choices as a Function of Temporal Proximity and Frame for the Asian Disease Problem

\begin{tabular}{|c|c|c|c|c|}
\hline \multirow[b]{3}{*}{ Temporal proximity } & \multicolumn{4}{|c|}{ Choices } \\
\hline & \multicolumn{2}{|c|}{ Risk-Avoidant } & \multicolumn{2}{|c|}{ Risk-Seeking } \\
\hline & $N$ & $\%$ total & $N$ & $\%$ total \\
\hline \multicolumn{5}{|l|}{ One week } \\
\hline \multicolumn{5}{|l|}{ Frame: } \\
\hline Gains & 27 & $63 \%$ & 16 & $37 \%$ \\
\hline Losses & 19 & $44 \%$ & 24 & $56 \%$ \\
\hline \multicolumn{5}{|l|}{ One year } \\
\hline \multicolumn{5}{|l|}{ Frame: } \\
\hline Gains & 22 & $51 \%$ & 21 & $49 \%$ \\
\hline Losses & 12 & $28 \%$ & 31 & $72 \%$ \\
\hline \multicolumn{5}{|l|}{ Three years } \\
\hline \multicolumn{5}{|l|}{ Frame: } \\
\hline Gains & 27 & $63 \%$ & 16 & $37 \%$ \\
\hline Losses & 15 & $35 \%$ & 28 & $65 \%$ \\
\hline \multicolumn{5}{|l|}{ Thirty years } \\
\hline \multicolumn{5}{|l|}{ Frame: } \\
\hline Gains & 28 & $65 \%$ & 15 & $35 \%$ \\
\hline Losses & 11 & $26 \%$ & 32 & $74 \%$ \\
\hline
\end{tabular}

\section{METHOD}

Participants and Design. The study was composed of 152 participants, 75 females and 77 males. The design of our study included a 2 frame (success, fail) $\times 2$ temporal proximity (hours, years) betweensubjects factorial design.

Stimulus Materials: Framing Task. In this experiment, we used a different scenario to manipulate temporal proximity. We chose a risky-choice decision task developed by Levin et al. (2002). This 
task was adapted for our temporal proximity manipulation by the addition of one of the temporal indicators; "in several years" or "in several hours" (see parenthetical notations in the introduction below). In this task, participants were provided with the following introduction:

Imagine that one of your parents was diagnosed with having dangerously high levels of cholesterol and you are faced with choosing a treatment program (in several hours or in several years). Two programs have been developed for treating high levels of cholesterol. Assume that the following alternatives represent the exact estimates of the situation that you will be facing (in several hours or in several years).

Afterward, they were presented with the following two alternatives framed either as gains or losses (losses condition presented in parentheses):

If program A is adopted, two-thirds of the persons treated will succeed in reducing (fail to reduce) their cholesterol.

If program B is adopted, there is a one-third chance that none of the persons treated will succeed in reducing (fail to reduce) their cholesterol and a two-thirds chance that all of the persons will succeed in reducing (fail to reduce) their cholesterol.

Immediately afterwards, participants were asked to indicate their preference for the alternatives on a 7-point Likert scale where 1 indicated "Definitely would recommend A" and 7 represented "Definitely would recommend B."

Holistic Assessment Task. According to the analytic/holistic model, when decision-makers process a decision task using the holistic style they engage in contextual referencing. In contextual referencing, context cues are sought from the problem that will lead the decision-maker to initiate a framework and the problem is then evaluated within this framework. Because this process is dependent upon context cues (in our situation the frame), decisionmakers who are processing using the holistic style should have more elaborate, in-depth use of the frame. Consequently, they should have better recall for the problem's frame. Conversely, individuals processing using the analytic style should be more sensitive to the numeric magnitude of the alternatives and have less in-depth processing of contextual elements (i.e., the frame). And as a result, they should perform more poorly when recalling the frame. Because our manipulation of "hours" was designed to induce the analytic style, we predict that in this condition individuals will be more prone to making errors when recalling the frame. And because the "years" condition was designed to induce the holistic style, individuals in this condition should have relatively better recall of the frame and make fewer errors in our assessment task. 
Because we wanted to assess participant's ability to correctly recall the frame, we constructed a "posttest" of the decision alternatives to ascertain whether participants could accurately report the frame of the alternatives they had just evaluated. As presented in the example below, the frame is imbedded within the alternatives creating three "frame-choice" situations. Participants were asked to choose the correct alternative for each of the three options.

\section{(Please circle one)}

If program A is adopted, one-third of the persons treated will (fail or succeed) in reducing their cholesterol.

If Program B is adopted, there is a one-third chance that all of the persons treated will (fail or succeed) and a two-thirds chance that none of the persons treated will (fail or succeed) in reducing their cholesterol.

Procedure. Participants were seated in groups with an average of 10 participants. They were briefly introduced to the experimenter and all participants agreed to participate. All participants were randomly assigned to the temporal proximity and framing conditions and presented with the cholesterol decision task. After completing the decision task, participants were asked to turn the material face down on the desk in front of them. They were then provided with our recall task designed to assess the extent of holistic processing. After completion of this material, participants were debriefed and released from the experiment.

\section{RESULTS AND DISCUSSION}

Table 3 depicts the preference means for the decision alternatives across both temporal proximity and framing conditions. Consistent with our hypothesis, there appears to be a markedly strong framing effect in the temporally distal condition (years) and no effect for framing in the temporally proximal condition (hours). In order to assess the overall effect of our manipulations, we first performed a regression analysis with frame (success, fail) and temporal proximity (hours, years) as our independent variables and participants' reported preference for the risk-averse and risk-seeking alternatives as our dependent variable. This analysis revealed a significant main effect for the problem framing $F(1$, $148)=4.19, p<.05$ as well as a significant Frame $\times$ Temporal proximity interaction $F(1,148)=5.6, p<.02$. This interaction supports our predictions for relatively stronger framing effects in the more distant temporal condition. 
TABLE 3. Average Preference for Risk-Avoidant and Risk-Seeking Choices as a Function of Temporal Proximity and Frame for the Cholesterol Task

\begin{tabular}{lcr}
\hline Temporal Proximity & $N$ & Average \\
\hline Several hours & & \\
Frame: & 38 & 3.68 \\
$\quad$ Gains (succeed) & 38 & 3.6 \\
$\quad$ Losses (fail) & & \\
Several years & & 3.2 \\
Frame: & 38 & 4.3 \\
$\quad$ Gains (succeed) & 38 & \\
$\quad$ Losses (fail) & & \\
& &
\end{tabular}

We then wanted to examine the framing effect in each of our temporal proximity conditions. In order to investigate this we performed paired contrasts on each of these conditions. This analysis revealed that when the framing situation was presented as occurring in close temporal proximity (several hours), the gain/loss framing manipulation produced no significant framing effect $F<1$. However, when the framing situation was presented as occurring in relatively distant temporal proximity, a strong framing effect occurred $F(1,148)=9.7, p<.01$.

TABLE 4. Number of Correct and Incorrect Frame Recall Responses for Each Recall Question

\begin{tabular}{|c|c|c|c|c|}
\hline & \multicolumn{2}{|c|}{ Correct } & \multicolumn{2}{|c|}{ Incorrect } \\
\hline & $N$ & $\%$ total & $N$ & $\%$ total \\
\hline \multicolumn{5}{|c|}{ Question 1} \\
\hline Hours & 55 & 72 & 21 & 28 \\
\hline Years & 68 & 90 & 8 & 10 \\
\hline \multicolumn{5}{|c|}{ Question 2} \\
\hline Hours & 41 & 54 & 35 & 46 \\
\hline Years & 47 & 62 & 29 & 38 \\
\hline \multicolumn{5}{|c|}{ Question 3} \\
\hline Hours & 40 & 53 & 36 & 47 \\
\hline Years & 44 & 58 & 32 & 42 \\
\hline
\end{tabular}

A second aspect of our study was to examine possible evidence for holistic processing. The measure that we designed allowed us to assess individual participant's recall of the framing manipulation. The findings from this recall task are presented in Table 4. Recall that, according to the analytic/holistic model, when an individual processes decisions in the holistic style they engage in contextual referencing. Acentral aspect of this process is that contextual cues are sought and then relied upon for subsequent processing. 
Therefore, it follows that individuals processing with this style should be more apt to correctly recall the frame because it was more thoroughly used in the decision-making process and should be more salient to them. To investigate this possibility we performed a repeated measures analysis with temporal proximity (hours, years) acting as our independent variable and participants correct/incorrect recall of the three frame responses as our dependent variable. This analysis revealed a significant effect for temporal proximity $F(1,150)=3.9, p<.05$. As may be seen in Table 4 , participants in the distant temporal condition (years) were more accurate in reporting the problem frame than participants in the more proximal condition (hours). This finding provides support for our contention that individuals processed holistically in the relatively distant temporal framing condition.

\section{GENERAL DISCUSSION}

An inevitable part of any decision we face is that it will occur at some point in the future, be it near or far. In this paper, we examined how the temporal proximity of the event affected participants' decisions for a risky-choice decision task. Based upon the analytic/holistic model, we predicted a temporal/relevance hypothesis for temporal proximity and framing effects. We reasoned that when an event is presented as occurring in relatively close temporal proximity, it will be perceived as especially relevant. Consequently, individuals should process the decision with the more effortful analytic style leading to attenuated framing effects. When the event is expected to occur in the distant future, however, it should be relatively irrelevant and a holistic style of processing should be used, leading to pronounced framing effects.

The results support this temporal/relevance hypothesis. In Study 1 , events that were expected in the near future were seen as more relevant that those that were not expected until the distant future. In Study 2, we manipulated the temporal proximity of the widely tested Asian disease problem whereas in Study 3, we manipulated a different type of decision problem involving cholesterol treatment. Overall, the results provide support for our contention that framing effects will be attenuated when the event is expected to occur in relatively close temporal proximity.

Overall, our findings provide more insight into the underlying processes involved in a risky-choice framing task. Specifically, one aspect of the event in question, temporal proximity, affects how the decision alternatives are processed and the style of processing invoked will influence reliance on either the frame or numeric magnitude of the alternatives. 
Our findings highlight the need to consider contextual factors, including temporal proximity, which may influence the decisionmaking process. Prior research has identified other contextual factors that influence the decision process. For example, researchers have identified how the "arena" or situational aspects of the task may influence subsequent decisions. In one study, Fagley and Miller (1997) found that tasks involving human lives produced framing effects whereas tasks involving money did not. Similar findings by Schneider (1992) revealed that tasks presented in different situations invoke different levels of aspiration that in turn, influence the likelihood of framing. Further, Wang, Simons, and Brédart (2001) found that familial relationship to the target of the task (level of kinship) influenced the likelihood of framing effects. Taken together, we believe that the contextual presentation of the alternatives effects subsequent reliance on the frame. We present one aspect of the context, temporal proximity, that will affect processing and reliance on the decision frame. Accounting for these contextual factors should lead to a better picture of the decision-making process in future framing research.

\section{IMPLICATIONS AND FUTURE RESEARCH}

An interesting question for future research would be to examine how the event within the task (e.g., Asian disease, cholesterol treatment) may influence time imposition. Our pilot study revealed that the Asian disease had a great deal of variability for participants' estimates of when they expected it to occur, with an average of several years. It may be the case that some events are expected to be relatively close or relatively distant in temporal proximity. Generalizing from the results of our studies, this time imposition may make it more or less likely for framing effects to emerge in these different scenarios. This finding could account for some of the discrepant findings across the different arenas of task presentation (e.g., Bloomfield, 2006; Fagley \& Miller, 1997; Kühberger, 1998; Schneider, 1992).

As can be seen in Table 4, all three of the frame-recall opportunities produced more incorrect responses for hours than years. However, the first recall question appears to carry a majority of the influence. This finding suggests a type of primacy effect for the frame recall in holistic processing. While this is speculative, it does present an interesting question for future research. Also, recent research investigating the influence of alignable and nonalignable differences in choice options provides an interesting contrast for our results and future research. This research originates from early work by Tversky (1977) and later Structural Alignment theory (see for review Genter \& Markman, 1997). The primary focus of this research is whether decision-makers rely 
upon elements of the decision options that are present in each option (alignability) or elements not present in every option (nonalignability). In a series of studies, Zhang and Markman (2001) investigated how motivation influenced decision-makers reliance upon alignable vs. non-alignable elements. They found that when individuals where motivated in a task they tended to rely more upon non-alignable elements whereas they relied upon alignable elements when they were low in motivation.

More recent research has investigated how temporal factors may influence reliance upon alignable vs. nonalignable elements. Studies by Malkoc et al. (2005) examined alignability/ nonalignability reliance in light of temporal construal theory. They found that when a decision task was presented in the more distant future, decision-makers relied more upon nonalignable elements of the options whereas when the task was presented as occurring in the near future they relied more upon alignable elements.

It would seem that in a typical risky-choice framing task, elements of the frame (lives saved or people die) should be alignable elements of the choices. Consistent with the findings of Zhang and Markman (2001), we found that under high motivation (relevance) individuals relied less on the frame whereas under low motivation (relevance) they did consider the frame more. Different from Malkoc et al. (2005), we found that when decision-makers evaluated events occurring in the more distant future, they relied more upon alignable elements of the decision choice (i.e., the frame) and they appear to rely upon nonalignable elements when the event is to occur in the near future.

While our studies were not designed to ascertain alignability/ nonalignability reliance, these findings provide an interesting comparison. One appears supportive while the other seems contradictory. Reconciliation for this apparent discrepancy may be found in examining questions such as whether analytic information can be perceived as an alignable element of the task or whether the goals of the task may encourage one form of reliance as being superior to the other. Specifically, it may be the case that the goal of the task (e.g., increase or decrease) may influence whether individuals tend to rely upon alignable or nonalignable elements. Future research should attempt to address these types of questions with a task more specifically designed for these concerns. 


\section{NOTE}

1. These temporal points were determined by pilot testing. Because we wanted to maintain a realistic time frame for the events occurrence we asked a separate group of 111 participants to estimate when they believed the Asian disease would occur. The conditions in this study represent the mean and mode as well as the high and low range obtained from our question of temporal estimation for the Asian disease occurrence.

\begin{tabular}{|c|c|c|}
\hline Problem & Gains & Losses \\
\hline $\begin{array}{l}\text { Imagine that the US. is prepar- } \\
\text { ing for the outbreak of an un- } \\
\text { usual Asian disease, which is } \\
\text { expected to kill } 600 \text { people. } \\
\text { Two alternative programs to } \\
\text { combat the disease have been } \\
\text { proposed. Assume that the } \\
\text { exact scientific estimates of } \\
\text { the consequences of the pro- } \\
\text { grams are as follows: }\end{array}$ & $\begin{array}{l}\text { If program } A \text { is adopted, } 200 \\
\text { people will be saved. } \\
\text { If Program B is adopted, there is } \\
\text { a one-third probability that } \\
600 \text { people will be saved and } \\
\text { a two-thirds probability that } \\
\text { no people will be saved. }\end{array}$ & $\begin{array}{l}\text { If program } A \text { is adopted, } 400 \\
\text { people will die. } \\
\text { If Program B is adopted, there is } \\
\text { a one-third probability that } \\
\text { nobody will die and a } \\
\text { two-thirds probability that } \\
600 \text { people will die. }\end{array}$ \\
\hline
\end{tabular}




\section{REFERENCES}

Bartels, D. M. (2006). Proportion dominance: The generality and variability of favoring relative savings over absolute savings. Organizational Behavior and Human Decision Processes, 100, 76-95.

Bless, H., Betsch, T.,\&Franzen, A. (1998). Framing the framing effect: The impact of context cues on solutions to the Asian disease problem. European Journal of Social Psychology, 28, 287-291.

Bloomfield, A. N. (2006).Groupsize and the framing effect: Threats to human beings and animals. Memory \& Cognition, 34, 929-937.

Brownell, H.H., Pincus, D., Blum, A., Rehak, A. \& Winner, E. (1997). The effects of right-hemisphere brain damage on patients (use of terms of personal reference). Brain and Language, 57, 60-79.

Chaiken, S. (1987). The heuristic model of persuasion. In M.P. Zanna, J.M. Olson, \& C.P. Herman (Eds.), Social influence: The Ontario symposium (vol. 5, pp. 3-39). Hillsdale, NJ: Erlbaum.

Chaiken, S., \& Trope, Y. (1999). Dual-process theories in social psychology. New York: Guilford Press.

Chandran, S.,\& Menon, G. (2004).When a day means more than a year: Effects of temporal framing on judgments of health risk. Journal of Consumer Research, 31, 375-389.

Chatterjee, S., Heath, T.B., Milberg, S.J., \& France, K. R. (2000). The differential processing of price in gains and losses: The effects of frame and need for cognition. Journal of Behavioral Decision Making, 13, 61-75.

Curseu, P. L. (2006). Need for cognition and rationality in decision-making. Studia Psychologica, 48, 141-156.

Epstein, S., Lipson, A., Holstein, C.,\& Huh, E. (1992). Irrational reactions to negative outcomes: Evidence for two conceptual systems. Journal of Personality and Social Psychology, 38, 889-906.

Fagley, N.S., \& Miller, P.M. (1990). The effect of framing on choice: interactions with risk-taking propensity, cognitive style, and sex. Personality and Social Psychology Bulletin, 16, 496-510.

Fagley, N.S., \& Miller, P.M. (1997). Framing effects and arenas of choice: Your money or your life? Organizational Behavior and Human Decision Processes, 71, 355-373.

Gallagher, P., \& Dagenbach, D. (2007). Manipulating noise frequencies alters hemispheric contributions to decision making. Brain and Cognition, 64, 42-49. 
Genter, D., \& Markman, A.B. (1997). Structure mapping in analogy and similarity. American Psychologist, 52, 45-56.

Gilovich, T., Kerr, M., \& Medvec, V.H. (1993). Effect of temporal perspective on subjective confidence. Journal of Personality and Social Psychology, 64, 552-560.

Igou, E.R.\& Bless, H. (2007). On undesirable consequences of thinking: Framing effects as a function of substantive processing. Journal of Behavioral Decision Making, 20, 125-142.

Kahneman, D.,\& Tversky, A. (1979). Prospect theory: an analysis of decision under risk. Econometrica, 47, 263-291.

Kühberger, A. (1998). The influence of framing on risky decisions: A meta-analysis. Organizational Behavior and Human Decision Processes, 57, 23-55.

Lauriola, M.,\& Levin, I.P. (2001). Personality traits and risky decision-making in a controlled experimental task: An exploratory study. Personality and Individual Differences, 31, 215-226.

LeBoeuf, R.A., \& Shafir, E. (2003). Deep thoughts and shallow frames: On the susceptibility to framing effects. Journal of Behavioral Decision Making, 16, 77-92.

Leiberman, N., Sagristano, M.,\&Trope, Y. (2002). The effect of temporal distance on level of construal. Journal of Experimental Social Psychology, 38, 523-535.

Leny-Meyers, J., \& Maheswaran, D. (2004). Exploring message framing outcomes when systematic, heuristic or both types of processing occur. Journal of Consumer Psychology, 14, 159-167.

Levin, I. P.,\& Chapman, D.P. (1990). Risk taking, frame of reference, and characterization of victim groups in AIDS treatment decisions. Journal of Experimental Social Psychology, 26, 421-434.

Levin, I.P., Gaeth, G.J., Schneider, S.L.,\& Lauriola, M. (2002). A newlook at framing effects: Distribution of effect sizes, individual differences, and independence of types of framing. Organizational Behavior and Human Decision Processes, 88, 411-429.

Levin, I. P., Schneider, S.L.,\& Gaeth, G.J. (1998). All frames are not created equal: A typology and critical analysis of framing effects. Organizational Behavior \& Human Decision Processes, 76, 149-188.

Liberman, N.,\& Trope, Y. (1998). The role of feasibility and desirability considerations in near and distant future decisions: A test of temporal construal theory. Journal of Personality and Social Psychology, 75, 5-18.

Machina, M.J. (1982). "Expected Utility" Analysis without the independence axiom. Econometrica, 50, 277-323. 
Malkoc, S. A., Zauberman, G.,\&Ulu, C. (2005). Consuming now or later? The interactive effect of timing and attribute alignability. Psychological Science,

16, 411-417.

McElroy, T.,\& Seta, J. J. (2003). Framing effects: Ananalytic-holistic perspective. Journal of Experimental Social Psychology, 39, 610-617.

McElroy, T.,\& Seta, J. J. (2004). On the other hand aml rational? Hemispheric activation and the framing effect. Brain and Cognition, 55, 572-580. (Reprinted in Complexity Digest issue 2004.28).

McElroy, T., \& Seta, J. J. (2006). Does it matter if it involves my group? How the importance of collective-esteem influences a group-based framing task.

Social Cognition, 24, 319-333.

McElroy, T., \& Seta, J. J., Warring, D. (in press). Reflections of the self: How self-esteem determines decision framing and increases risk-taking. Journal of Behavioral Decision Making.

Miller, P.M., \& Fagley, N.S. (1991). The effects of framing, problem variations, and providing rationale on choice. Personality and Social Psychology Bulletin, 17, 517-522.

Petty, R.E.,\& Cacioppo, J.T. (1986). Communication and persuasion: Central and peripheral routes to attitude change. New York: Springer-Verlag.

Reyna, V.F., \& Brainerd, C.J. (1991). Fuzzy-trace theory and framing effects in choice: Gist extraction, truncation, and conversion. Journal of Behavioral Decision Making, 4, 249-262.

Sanna, L. J., \& Chang, E. C. (2006). Judgments over time: The interplay of thoughts, feelings, and behaviors. New York: Oxford University Press.

Sanna, L. J., Chang, E. C., Parks, C. D.,\& Carter, S. E. (2005). The hourglass is half full or half empty: Temporal framing and the group planning fallacy.

Group Dynamics: Theory, Research, and Practice, 9, 173-188.

Sanna, L.J., \& Schwarz, N. (2004). Integrating temporal biases: The interplay of focal thoughts and accessibility experiences. Psychological Science, 15, 474481.

Schneider, S. L. (1992). Framing and conflict: Aspiration level contingency, the status quo, and current theories of risky choice. Journal of Experimental Psychology: Learning, Memory, and Cognition, 18, 1040-1057.

Sieck, W., \& Yates, J.F. (1997). Exposition effects on decision making: choice and confidence in choice. Organizational Behavior and Human Decision Processes, 70, 207-219.

Simon, A. F., Fagley, N.S.,\& Halleran, J.G. (2004). Decision framing: Moderating effects of individual differences and cognitive processing. Journal of Behavioral Decision Making, 17, 77-93. 
Slovic, P.,\&Lichtenstein, S. (1968). Relative importance of probabilities and payoffs in risk taking. Journal of Experimental Psychology, 78, 1-17.

Smith, S. M., \& Levin, I.P. (1996). Need for cognition and choice in framing effects. Journal of Behavioral Decision Making, 9, 283-290.

Stanovich, K.E., \& West, R.F. (2000). Individual differences in reasoning: Implications for the rationality debate? Behavioral and Brain Sciences, 23, 645-726.

Takemura, K. (1994). Influence of elaboration of the framing of decision. The Journal of Psychology, 128, 33-39.

Trope, Y., \& Liberman, N. (2003). Temporal construal. Psychological Review, 110, 403-421.

Tversky, A. (1977). Features of similarity. Psychological Review, 84, 327-352. Tversky, A.,\& Kahneman, D. (1981). The framing of decisions and the rationality of choice. Science, 221, 453-458.

Wang, X.T. (2004). Self-framing of risky choice. Journal of Behavioral Decision Making, 17, 1-16.

Wang, X.T. (2006). Emotions within reason: Resolving conflicts in risk preference. Cognition and Emotion, 20, 1132-1152.

Wang, X.T., Simons, F., \& Bredart, S. (2001). Social cues and verbal framing in risky choice. Journal of Behavioral Decision Making, 14, 1-15.

Zhang, Y., \& Buda, R. (1999). Moderating effects of need for cognition on responses to positively versus negatively framed advertising messages.

Journal of Advertising, 28, 1-15.

Zhang, S., \& Markman, A. B. (2001). Processing product unique features:

Alignability and involvement in preference construction. Journal of Consumer Psychology, 11, 13-27. 